\title{
CAC Regimen
}

National Cancer Institute

\section{Source}

National Cancer Institute. CAC Regimen. NCI Thesaurus. Code C161942.

A chemotherapy regimen consisting of carboplatin, cyclophosphamide and doxorubicin

that may be used in the treatment of breast and ovarian cancers. 